\title{
Image Preprocessing Methods to Identify Micro-cracks of Road Pavement
}

\author{
Hui Wang, Zhang Chen ${ }^{*}$, Lijun Sun \\ Key Laboratory of Road and Traffic Engineering of the Ministry of Education, Tongji University, Shanghai, China \\ Email: mickysophy@163.com
}

Received 2013

\begin{abstract}
Standards of highway conservation and maintenance are improved gradually following the improvement of requirements of road service. Before obvious damage such as obvious cracking (block, transverse, longitudinal ) and rutting emerge, inconspicuous distress (micro-cracks, polishing, pockmarked) is generated previously. These inconspicuous distresses may provide basis and criteria for pavement preventive maintenance. Currently most of preventive conservation measures are determined by experienced experts in maintenance and repair of road after site visits. Thus method is difficult in operation, and has a certain amount of instability as it is based on experience and personal knowledge. In this paper, camera and laser were used for automated high-speed acquisition images. Methods to preprocess pavement image are compared. The pretreatment method suitable for analyze micro-cracks picture is elected, an effective way to remove shadow is also proposed.
\end{abstract}

Keywords: Pavement Distress Automatic Detection; Inconspicuous Distress; Micro-crack; Laser Light Image; Image-preprocessing

\section{Introduction}

Preventive maintenance should be integrated into pavement manage system to determine the proper time for operation. The U.S. scholar Zimmennan K.A had prospected (2003), "transportation agencies want to combine preventive maintenance and pavement management, which requires pavement condition surveys and calculation of indicators".

Inconspicuous distresses (micro-cracks, polishing, pockmarked) are generated previously before obvious distress generates. These distresses are difficult to be found and recognized, especially micro-cracks (millimeter level), developed from isolation of aggregates of road surface. Polishing and pockmarked are difficult to be quantified itself, traditional recognized human vision, but a manual surface distress survey is subjected to many limitations, such as not repeatable, too subjective and high human cost.

Line laser light and camera has been used for detection rutting by calculating the curve changes of the line (Yuntao Wei etc. 2009).

In this paper, a laser line and a camera is used to detect micro-cracks and its width. Image processing method is descripted below.

\section{Background Correction}

The composition of the road pavement image is mainly generated by three aspects. One is background lighting signal, including shadow information. One is noise signal, mainly caused by the road surface texture. Last one is distress signal, which consists of pavement distress, stains, marking.

The purpose of image background correction is to exclude the background interference brought by light intensity uneven. Pavement distress information should be assured during this process. The whole image matrix is made into small matrix of the same size, using matrix grayscale characteristics of the image background, image relative ideal background is calculated and analyzed. Reference background method is used to correct image background, results are shown in Figure 2-1, Figure 2-2.

\section{Image Noise Reduction and Image Enhance}

Noise caused by background non uniform disappeared by background correction, but still contains a small amount of random noise, which needs further eliminate. Noise reduction effect by 10 different noise-reduction methods is shown in Figure2-3.

1, Original image; 2, Low-pass convolution template, suppose original image $f(\mathrm{LI}), h(h, h)$ is impulse response of low-pass filtering( $\mathrm{L} \times \mathrm{L}$ matrix),

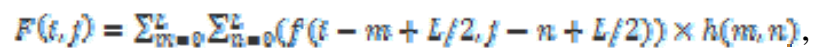




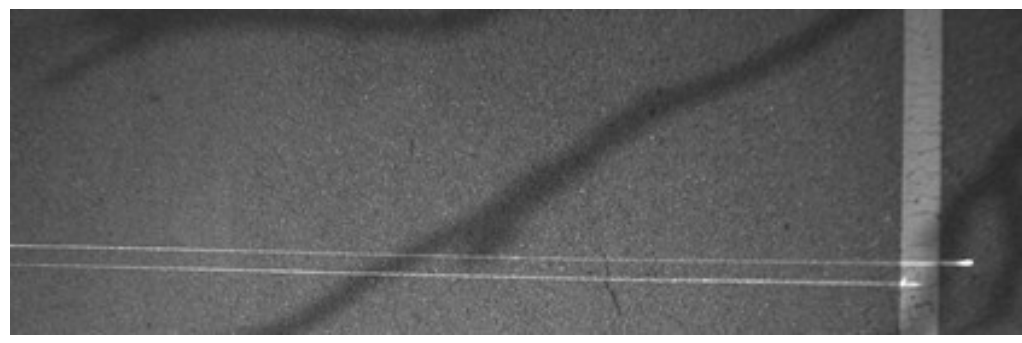

Original image

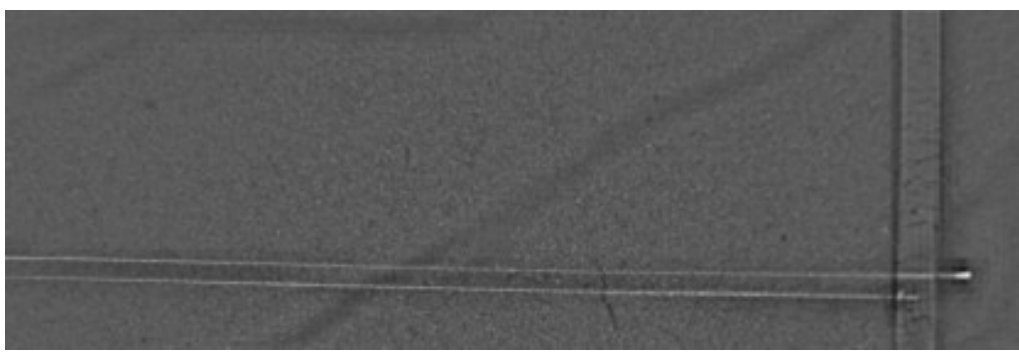

After background-correction

Figure 2-1 Road pavement image.
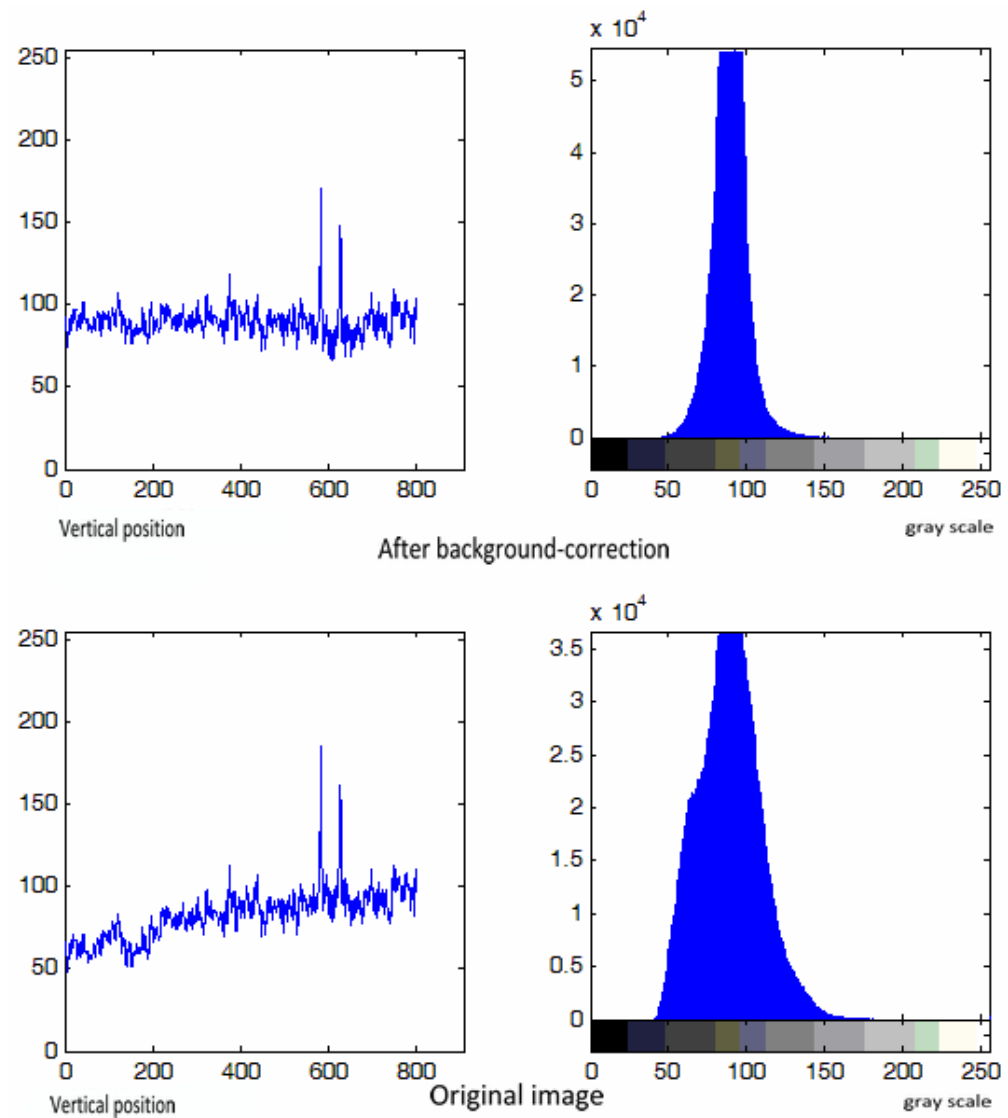

Figure 2-2 Gray values and Gray histogram of road pavement image.

the template is "Box template", $\mathrm{h}=\frac{1}{(}\left(\begin{array}{lll}1 & 1 & 1 \\ 1 & 1 & 1 \\ 1 & 1 & 1\end{array}\right) ; 3, \quad$ High-pass filtering, $F_{1}=\left(\begin{array}{ccc}0 & -1 & 0 \\ -1 & 5 & -1 \\ 0 & -1 & 0\end{array}\right) ; 4$, 
High-pass filtering, $K_{\mathrm{z}}=\left(\begin{array}{ccc}-1 & -1 & -1 \\ -1 & 9 & -1 \\ -1 & -1 & -1\end{array}\right) ; 5$,

High-pass filtering, $K_{g}=\left(\begin{array}{ccc}1 & -2 & 1 \\ -2 & 5 & -2 \\ 1 & -2 & 1\end{array}\right) ; 6$,

High-pass filter, $K_{4}=\frac{1}{7}\left(\begin{array}{ccc}1 & -2 & 1 \\ -2 & 19 & -2 \\ 1 & -2 & 1\end{array}\right) ; 7$, High-pass

filtering, $K_{\mathrm{g}}=\frac{1}{2}\left(\begin{array}{ccc}1 & -2 & 1 \\ -2 & 0 & -2 \\ 1 & -2 & 1\end{array}\right) ; 8$, Mean Filtering; 9,

Threshold filtering; 10, Median filtering It can be seen through Figure2-4, method 2 and method 7 are better.
However, road distress signal is very weak, therefore distress information should be assured, enhancing distress information, weakening background information.

\section{Distress Image Segmentation}

There are lots of methods to obtain optimal threshold, such as range method, the largest category of variance method. In this paper, region without distress is separated from calculation, then range method is used, result is shown in Figure2-5.

\section{Conclusions}

By analyzing the gradation characteristics of the pavement micro-cracks image, background correction method suitable for this type of image is selected, also effect of the shadow is effectively eliminated.
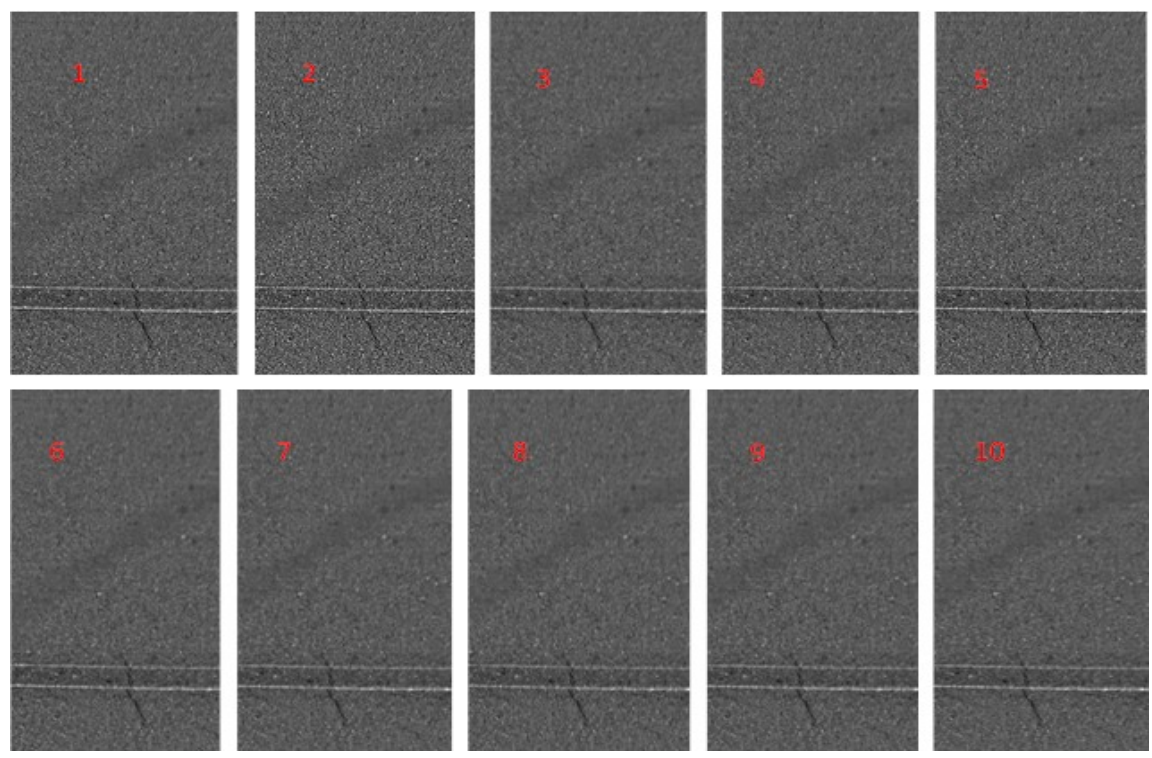

Figure 2-3. Images after different enhance methods.
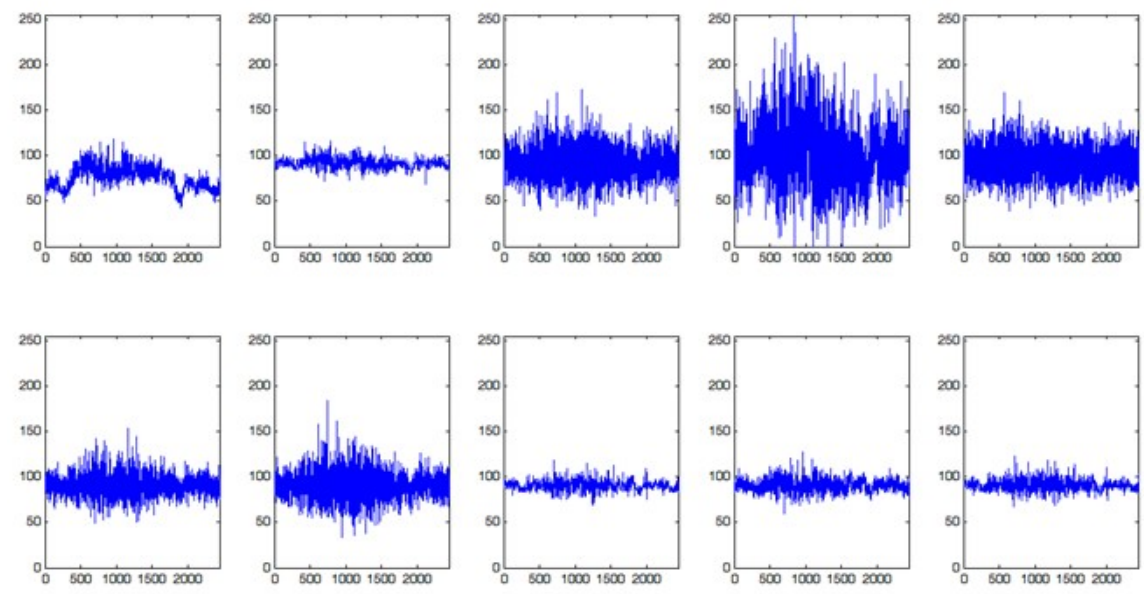

Figure 2-4. Gray values of road pavement image. 


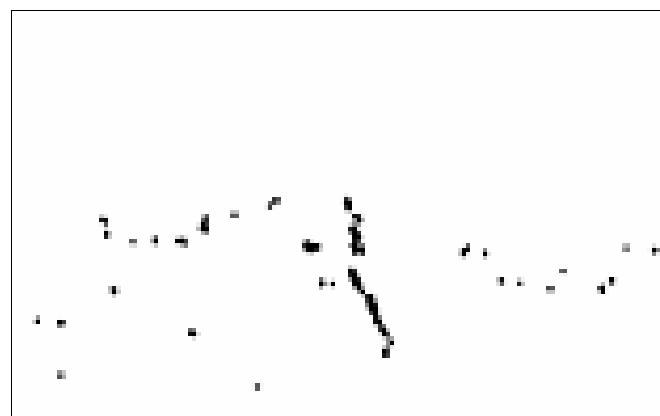

Figure 2-5. Image segmentation of road pavement image.

On the basis of the background correction, several noise deduction methods are compared. Low-pass convolution template method is better.

Finally, distress image is segmented.

\section{REFERENCES}

[1] K. A. Zimmerman, "Pavement Management Perspective and Integrating Preventive Maintenance into A Pavement Management System,” Transportation Research Record, No.1827, 2003, pp. 3-9.

[2] J. N. Meegoda, S.Y. Gao, S. Liu and N. C. Gephart, "Pavement Texture from High-Speed Laser for Pavement Management System,” International Journal of Pavement Engineering, 2012, pp.1-9.

[3] M.-T. Do, Z. Z. Tang, M. Kane, Francois de Larrard, "Pavement Polishing-Development of A Dedicated Laboratory Test and Its Correlation with Road Results," Wear, No. 263, 2007, pp. 36-42.

[4] Y.T. Wei, H.Y. Hong, X. H. Zhang and J. Y. Yu, “A New Method for Automatic Detection of Rut Feature Based on Road Laser Images,” Multispectral Image Acquisition and Processing, Proceedings of SPIE, Vol. 7494, 2009. 